\title{
Convective processes and hydromagnetic instabilities in core collapse supernova simulations
}

\author{
M. Obergaulinger ${ }^{1}$, N. J. Hammer ${ }^{1}$ and E. Müller ${ }^{1}$ \\ ${ }^{1}$ Max-Planck-Institut für Astrophysik, Karl-Schwarzschildstr. 1, 85741 Garching, Germany \\ email: mobergau@mpa-garching.mpg.de, hammer@mpa-garching.mpg.de
}

\begin{abstract}
Hydrodynamic and potentially also hydromagnetic instabilities play an important role in core-collapse supernovae. We report on simulations performed to investigate the effects of magnetic fields in simplified models. Furthermore, we present efforts to get rid of problems arising from the coordinate singularity at the symmetry axis and the associated boundary conditions.
\end{abstract}

Keywords. Hydrodynamics, MHD, supernovae: general, methods: numerical

\section{MHD simulations of stellar core collapse}

Convection and further hydrodynamic instabilities are of key importance for reviving the stalled shock inside the core of a type-II supernova explosion (Janka (2001)). Detailed simulations (e.g. Buras et al. (2006)) show the development of Ledoux and Schwarzschild convection in the nascent proto-neutron star and the ambient hot-bubble region. Furthermore, hydrodynamic instabilities such as the advective-acoustic cycle have been discussed as an explanation for the global asphericity of supernova explosions and the associated large kick velocities of neutron stars (Galletti \& Foglizzo (2005), Scheck et al. (2006)).

Additionally, magnetic fields may modify the dynamics of the explosion and the structure of the remnant neutron star. Possible effects of magnetic fields (Wheeler et al. (2005)), once amplified sufficiently, are the enhanced transport of angular momentum, the creation of bipolar jet-like outflows, or an impact on the microphysics of the stellar gas and its interaction with neutrino radiation. In certain cases, even explosions driven mainly by magnetic fields are conceivable.

Taking into account the uncertainties still existing concerning the rotation and magnetic field of a stellar pre-collapse core, we performed a comprehensive parameter study of the magneto-rotational collapse of stellar cores to proto neutron stars in axisymmetry employing a newly developed numerical code (Obergaulinger et al. (2006a), Obergaulinger et al. (2006b)). We greatly simplified the physics involved in the explosion by approximating the complex nuclear equation of state by an analytic model, by entirely neglecting neutrino interactions and transport, and by considering simplified equilibrium initial models. These simplifications make our models applicable only to the first stages of the explosion. We started with a purely poloidal initial field centered around a current loop in the equatorial plane.

During collapse, the poloidal field is amplified due to flux-conserving compression, and as the cores rotate increasingly fast and differential, a strong toroidal field component is created from the initially purely poloidal one (see Fig. 1, left panel). A large amount of the rotational energy can be used building up a magnetic field of complex structure. Due to the axisymmetry assumed, the conversion of toroidal to poloidal fields and hence a full dynamo are suppressed. 

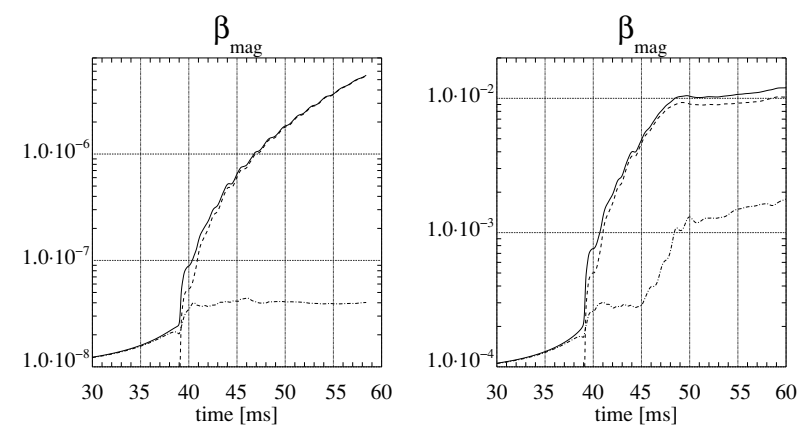

Figure 1. Evolution of the ratio of magnetic energy to gravitational energy, $\beta_{\mathrm{mag}}=E_{\mathrm{mag}} /\left|E_{\text {grav }}\right|$ of two cores. The solid, dashed, and dashed-dotted lines show the energy of the total field and the toroidal and poloidal components, respectively. The model depicted in the left panel exhibits no MRI-like modes, i.e. the poloidal field stays constant after the collapse (after $t \sim 39 \mathrm{~ms}$ ). The poloidal field shown in the right panel grows due to MRI modes exponentially $(t \sim 45 . .50 \mathrm{~ms})$.

Additional amplification can occur via convective processes or the magneto-rotational instability. Potentially, a dynamo can operate under these conditions. In principle, most of our models are prone to MRI because of the strongly differential rotation in the postcollapse core. To study the instability numerically is, however, not trivial due to the limited grid resolution. Nevertheless, several models exhibit signs of field amplification due to MRI. In these cases, the energy of the poloidal field grows exponentially (see Fig. 1, right panel), and efficient angular momentum transport sets in. The extraction of centrifugal support consequently alters the structure of the core. In addition, bipolar outflows are generated by the magnetic field in such models.

In a number of models, we imposed an extremely strong initial field, which, even without any instability-related amplification, affects the stability of the core and the dynamics of the explosion. Angular momentum transport as well as the emergence of jet-like outflows can be observed. The outflows resemble the typical structure of jets collimated by magnetic fields with an internal compressing part ("beam") surrounded by an external expanding "cocoon". Such jets are the source of a unique gravitational wave signal, which would allow their identification in a galactic supernova by current detectors.

\section{Axis-free combined grid methods}

Since stars have a basically spherical symmetric shape, it is natural to choose spherical polar coordinates when performing multi-D hydrodynamic simulations of their evolution. Although spherical grids have several advantages, such as the possibility to choose a logarithmically spaced radial grid, one has to face the fact that numerical problems may arise from the coordinate singularity and the boundary conditions at the polar symmetry axis. In addition, the meridians converging towards the polar singularity lead to a restrictive CFL condition at the axis, which makes especially 3D simulations computationally very expensive and one often is forced to cut out a conical region embedding the axis to reduce the computational costs.

An example for numerical artefacts created by the symmetry axis is shown in Fig. 2, which was taken from Kifonidis et al. (2006). At that time the flow has developed a jetlike feature, which is located along the polar symmetry axis. There is no physical reason for this feature. Instead, it is a numerical artefact produced most likely by the reflecting boundary condition at the axis, and hence the impossibility to transport momentum 


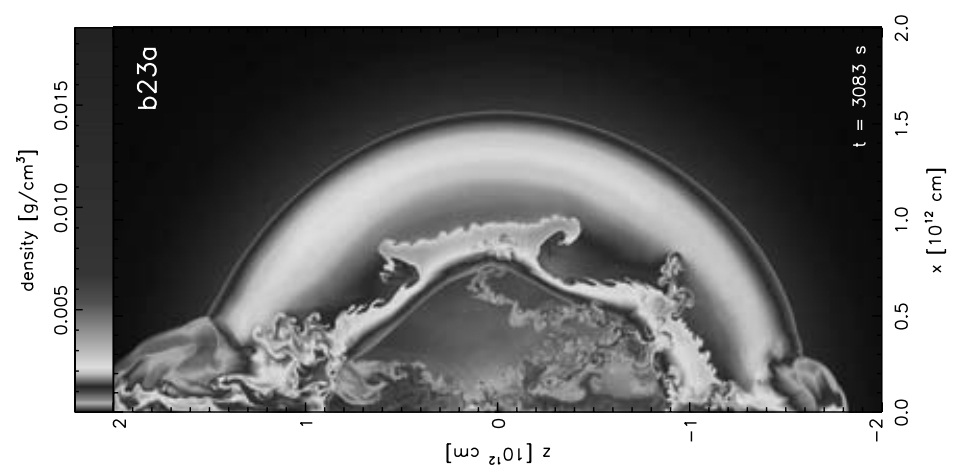

Figure 2. Snapshot from a 2D long-term simulation performed by Kifonidis et al. (2006), showing the density structure of a $15 \mathrm{M}_{\odot}$ supernova progenitor's stellar envelope roughly 50 min after core collapse, formed by a cascade of various hydrodynamic instabilities, e.g. Rayleigh-Taylor and Richtmyr-Meshkov instabilities. One can clearly see the dense, jet-like structures along the symmetry axis' boundary, which have already broken through the explosion shock wave, whereas all structures sufficiantly far from the axis are correctly located well behind the shock front.

across the axis. The feature spoils the simulation results in roughly 20 percent of the angular computational domain centered around the axis. The situation is even worse at later epochs.

To avoid these severe problems, which occur conspicuously in longterm simulations, utilising spherical coordinates we are developing an axis-free multi-patch spherical grid method. Currently we are performing extensive test calculations with the new method.

\section{Future developments}

After this first still preliminary investigation, we plan to improve the approximations decribing various physical effects during collapse and explosion. This requires the inclusion of a more realistic microphysical equation of state and of an approximative neutrino transport to our MHD code.

The new grid technology will be of use for various applications in the context of core collapse supernovae simulations. For example, we plan to apply the new grid in GRMHD simulations with 3 spatial dimensions, where one expects jet features, which may be spoiled by axis effects.

\section{Acknowledgements}

M.O. wants to thank the IAU for providing financial support by an IAU travel grant.

\section{References}

Buras, R., Rampp, M., Janka, H.-Th., \& Kifonidis, K. 2006, A $\& A 447,1024$

Galletti, P. \& Foglizzo, T. 2005, in Proc. of the SF2A meeting, 17-30 June 2005, Strasbourg, EDPS Conf. Series in $A \& A A$ eds. F. Casoli, T. Contini, J.M. Hameury, \& L. Pagani Janka, H.-Th. 2001, A\&\&A 368, 527

Kifonidis, K., Plewa, T., Scheck L., Janka, H.-Th., \& Müller, E. 2006, A\&A 453, 661

Obergaulinger, M., Aloy, M.A., Dimmelmeier, H., \& Müller, E. (2006b), A\& $A$ 457, 209

Obergaulinger, M., Aloy, M.A., \& Müller, E. (2006a), A\&SA 450, 1107

Scheck, L., Kifonidis, K., Janka, H.-Th., \& Müller, E. (2006) ArXiv Astrophysics e-prints

Wheeler, J. C. \& Akiyama (2005) in Open issues in core collapse supernova theory, Proceedings from the Institute for Nuclear Theory, World Scientific Publishing Company, eds. A. Mezzacappa, \& G.M. Fuller 